\title{
Maximum likelihood estimation for germination-growth processes with application to neurotransmitters data
}

\author{
S. N. Chiu ${ }^{1, *}$ I. S. Molchanov, ${ }^{2}$ and M. P. Quine, ${ }^{3}$ \\ ${ }^{1}$ Department of Mathematics, Hong Kong Baptist University, Kowloon, Hong Kong \\ ${ }^{2}$ Department of Statistics, University of Glasgow, Glasgow G12 8QW, UK \\ ${ }^{3}$ School of Mathematics and Statistics, University of Sydney, N. S. W. 2006, Australia
}

A maximum likelihood procedure is given for estimating parameters in a germination-growth process, based on germination times only or on both times and locations. The process is assumed to be driven by a Poisson process whose intensity is of known analytical form. The procedure is shown to perform well on simulated data with unnormalised gamma intensity and is also applied to data on release of neurotransmitter at a synapse.

Keywords: Germination-growth; Maximum likelihood estimation; Neurobiology; Poisson; Synapse.

${ }^{*}$ Corresponding author: E-mail: snchiu@math.hkbu.edu.hk 


\section{Introduction}

A maximum likelihood estimation procedure is proposed for analysing data of autoinhibited release of neurotransmitters at a synapse. The mechanism has been suggested by Bennett and Robinson (1990) and is as follows. The terminal of a neuronal axon at the neuromuscular junction has branches consisting of strands containing many randomly scattered sites. At a synapse an action potential triggers the release of neurotransmitter at these sites. Each quantum released is assumed to cause release of an inhibitory substance which diffuses along the terminal at a constant rate preventing further releases in the inhibited region. Thus, some potential releases have been prohibited. The aim of the current study is to estimate the value of the constant rate of the diffusion of inhibitory substance and the intensity of potential releases based on the measurement of times of actual release of transmitters.

The data sets that can be accessed at

http://www . maths. usyd.edu . au:8000/u/malcolmq/

contain the times and the amplitudes of release of all transmitters in a series of 800 experiments provided by Professor M. R. Bennett of the Neurobiology Research Centre at the University of Sydney. The number of releases ranges from 0 to 4 . The frequencies of 0's, 1's, . . is 101, 387, 237, 66, 9. These figures are very similar to the results of another series of 800 experiments given as Table 2 of Quine and Robinson (1992).

These data have been analysed by Chiu, Quine and Stewart (2000) and Molchanov and Chiu (2000). The former paper suggested that the amplitude of each release could be served as a surrogate for location, which was not observable, of release of each transmitter by taking an inverse power transformation distance $=1 / \sqrt{\text { amplitude }}$. The estimation method in Chiu et al. (2000) was based on both the times and locations of releases. Molchanov and Chiu (2000) studied the same problem and they estimated the intensity of potential releases 
nonparametrically based only on times of releases, provided that the rate of diffusion was known. However, they did not suggest a way to estimate the rate of diffusion based on release times only. In an earlier study, Quine and Robinson (1992) assumed that the intensity of potential releases is a constant and derived maximum likelihood estimators for this constant and the rate of diffusion by considering the time of the first release and of the second release, if there is one. However, this homogeneous model was oversimplified for the application and shown to be inadequate by data. The reason is that under the homogeneous model there should always be at least one release, but 101 out of 800 experiments contain no release.

In this paper the maximum likelihood estimation of a general parametrised intensity of potential releases and the rate of diffusion of inhibitory substance based on times only, and on both times and locations, will be studied. Unlike Quine and Robinson (1992), the estimation will take all observations into consideration.

\section{The model}

Consider a Poisson process $\Psi$ on $[0, W]^{d} \times[0, \infty)$ with intensity measure $\mathrm{d} \mathbf{x} \mathrm{d} \Lambda(t)$, where $\mathbf{x} \in[0, W]^{d}$ and $\Lambda$ is a non-decreasing function satisfying that $\Lambda(t)=0$ for $t<0, \Lambda(t)<\infty$ for $t<\infty$ and $\Lambda(\infty)>0$. Seeds start to germinate on $[0, W]^{d}$ at time zero according to $\Psi$. Once the seed $\left(\mathbf{x}_{i}, t_{i}\right)$ is germinated (at location $\mathbf{x}_{i}$ at time $t_{i}$ ), it starts generating an inhibiting area which is a sphere centred at $\mathbf{x}_{i}$ and growing radially in all directions with a constant speed $v$. New seeds continue to germinate on uninhibited parts of $[0, W]^{d}$ according

to $\Psi$ until the whole $[0, W]^{d}$ is inhibited. Throughout the paper $\Lambda$ is assumed to have a density function $\lambda$.

The releases of neurotransmitters at a synapse can be modelled well by the germination of seeds in the above germination-growth process in the case $d=1$. Moreover, the replication of a DNA molecule in higher animals (Vanderbei and Shepp, 1988; Cowan et al., 1995) and 
the differentiation of cells into heterocysts in algae (Wolk, 1975) can also be modelled by such a process with $d=1$. Such a germination-growth process was first suggested and studied for the case $d=2$ by Kolmogorov (1937) and Johnson and Mehl (1939) to model crystal growth, see Chiu (1995, 1997), Chiu and Quine (1997) and Okabe et al. (2000) for details of subsequent developments.

Quine and Robinson (1992) have estimated $\Lambda$ for the neurotransmitters data by assuming $\Lambda(t)=\alpha t$ for some positive finite $\alpha$, but this was oversimplified for the neurobiological data. A more realistic $\Lambda$ suggested in the literature for this application (e.g. Thomson et al., 1995) is

$$
\Lambda(t)=\alpha \int_{0}^{t} \frac{\gamma^{k}}{\Gamma(k)} s^{k-1} e^{-\gamma s} \mathrm{~d} s,
$$

where $\alpha, \gamma$ and $k$ are all positive and finite. Chiu et al. (2000) and Molchanov and Chiu (2000) estimated $\Lambda$ nonparametrically and the former paper got estimates for $\alpha, \gamma$ and $k$ by minimising the absolute deviations between the nonparametric estimate of $\Lambda$ and the parametrised form given in (2.1).

\section{Likelihood with observable locations and germination times}

Suppose there are $n$ independent realisations of the above germination-growth model according to $n$ independent Poisson processes $\Psi_{i}, 1 \leq i \leq n$, and the first $m$ of them are non-empty, i.e. they contain at least one germination. Denote by $\Phi_{i}=\left\{\left(\mathbf{x}_{i 1}, t_{i 1}\right), \ldots,\left(\mathbf{x}_{i n_{i}}, t_{i n_{i}}\right)\right\}$ the observed locations and germination times of the seeds in the $i$ th non-empty realisation, where $n_{i}$ is the number of germinations and $i=1, \ldots, m$. The likelihood of observing $\Phi_{i}$ inside $[0, W]^{d}$ is equal to the product of the likelihood $\prod_{\left(\mathbf{x}_{i j}, t_{i j}\right) \in \Phi_{i}} \lambda\left(t_{i j}\right)$ that Poisson points from $\Psi_{i}$ appear at the points of $\Phi_{i}$ and the probability $\exp \left\{-\int_{B_{i}(v)} \lambda(t) \mathrm{d} \mathbf{x} \mathrm{d} t\right\}$ that no other Poisson points from $\Psi_{i}$ apart from those appearing in $\Phi_{i}$ are germinated seeds, where $B_{i}(v)$ is the space-time region in $[0, W]^{d} \times[0, \infty)$ which lies beneath, with respect to the time axis, cones 
rising from each point in $\Phi_{i}$; the section of each cone at a height $t$ above the vertex is a $d$-dimensional ball of radius $v t$.

Thus, the likelihood of observing $n-m$ empty realisations and $\Phi_{i}, i=1, \ldots, m$, is

$$
L_{1}(\lambda, v)=\left[\exp \left\{-W^{d} \Lambda(\infty)\right\}\right]^{n-m} \prod_{i=1}^{m}\left[\left\{\prod_{\left(\mathbf{x}_{i j}, t_{i j}\right) \in \Phi_{i}} \lambda\left(t_{i j}\right)\right\} \exp \left\{-\int_{B_{i}(v)} \lambda(t) \mathrm{d} \mathbf{x} \mathrm{d} t\right\}\right] .
$$

The speed $v$ appears only in $B_{i}(v)$ and is such that $B_{i}\left(v_{1}\right) \subset B_{i}\left(v_{2}\right)$ whenever $v_{1}>v_{2}$, because the cones are bigger when $v$ increases. Thus, no matter what $\lambda$ is, $L_{1}(\lambda, v)$ increases with $v$ and so the maximum likelihood estimator $\widehat{v}$ of the speed is the largest possible $v$ such that none of the points in $\Phi_{i}$ is contained in the interior of a cone. If $\lambda$ is of known analytical form and with a finite number of unknown parameters, these parameters can be estimated by maximising $L_{1}(\lambda, \widehat{v})$.

Example 1. Consider that $\lambda(\cdot)=\alpha \mathbf{1}_{[a, b]}(\cdot)$ where $\alpha$ is a positive constant and $\mathbf{1}_{[a, b]}$ the indicator function of the interval $[a, b]$. Then

$$
\begin{aligned}
L_{1}(\lambda, v)=\left[\exp \left\{-W^{d} \alpha(b-a)\right\}\right]^{n-m} & \\
\times & \prod_{i=1}^{m}\left[\left\{\prod_{\left(\mathbf{x}_{i j}, t_{i j}\right) \in \Phi_{i}} \alpha \mathbf{1}_{[a, b]}\left(t_{i j}\right)\right\} \exp \left\{-\int_{B_{i}(v)} \alpha \mathbf{1}_{[a, b]}(t) \mathrm{d} \mathbf{x} \mathrm{d} t\right\}\right] .
\end{aligned}
$$

As mentioned above, the maximum likelihood estimator $\widehat{v}$ of the speed is the largest possible $v$ such that none of the points in $\Phi_{i}$ is contained in the interior of a cone. Moreover, $L_{1}(\lambda, v)$ is zero if some $t_{i j} \notin[a, b]$. Under the constraint that $a \leq \min \left(t_{i j}\right) \leq \max \left(t_{i j}\right) \leq b$, the likelihood $L_{1}(\lambda, v)$ increases if the difference $b-a$ decreases. Thus, no matter what the values $\alpha$ and $v$ take, the maximum likelihood estimators $\widehat{a}$ and $\widehat{b}$ of the parameters $a$ and $b$ are the minimum and the maximum of the observed germination times $\left\{t_{i j}: 1 \leq j \leq n_{i}, i=1, \ldots, m\right\}$, respectively. Then without loss of generality we can write

$$
L_{1}(\lambda, v)=\left[\exp \left\{-W^{d} \alpha(b-a)\right\}\right]^{n-m} \alpha^{\sum_{i=1}^{m} n_{i}} \exp \left\{-\sum_{i=1}^{m} \int_{B_{i}(v)} \alpha \mathbf{1}_{[a, b]}(t) \mathrm{d} \mathbf{x} \mathrm{d} t\right\} .
$$


Finally, consider

$$
\begin{aligned}
\frac{\partial \log L_{1}(\lambda, v)}{\partial \alpha} & =\frac{\sum_{i=1}^{m} n_{i}}{\alpha}-W^{d}(b-a)(n-m)-\sum_{i=1}^{m} \int_{B_{i}(v)} \mathbf{1}_{[a, b]}(t) \mathrm{d} \mathbf{x} \mathrm{d} t \\
\frac{\partial^{2} \log L_{1}(\lambda, v)}{\partial \alpha^{2}} & =-\frac{\sum_{i=1}^{m} n_{i}}{\alpha^{2}} .
\end{aligned}
$$

The maximum likelihood estimator of $\alpha$, therefore, is

$$
\widehat{\alpha}=\frac{\sum_{i=1}^{m} n_{i}}{W^{d}(\widehat{b}-\widehat{a})(n-m)+\sum_{i=1}^{m} \int_{B_{i}(v)} \mathbf{1}_{[\widehat{a}, \widehat{b}]}(t) \mathrm{d} \mathbf{x} \mathrm{d} t},
$$

with asymptotic variance $\widehat{\alpha}^{2} / \sum_{i=1}^{m} n_{i}$. The integral $\int_{B_{i}(v)} \mathbf{1}_{[\widehat{a}, \widehat{b}]}(t) \mathrm{d} \mathbf{x} \mathrm{d} t$ is simply the $(d+1)$ dimensional Lebesgue measure of the space-time region in $[0, W]^{d} \times[\widehat{a}, \widehat{b}]$ which is not covered by cones in the $i$ th nonempty realisation.

\section{Likelihood with observable germination times but unobservable locations}

In many applications, only the germination times but not the locations are observable. Nevertheless, if the underlying Poisson process is spatial homogeneous, maximum likelihood estimators can still be obtained. The likelihood of observing $n-m$ empty realisations and $m$ nonempty realisations of germination times $\left\{t_{11}, \ldots, t_{1 n_{1}}\right\}, \ldots,\left\{t_{m 1}, \ldots, t_{m, n_{m}}\right\}$ is

$$
\begin{aligned}
L_{2}(\lambda, v)=\left[\exp \left\{-W^{d} \Lambda(\infty)\right\}\right]^{n-m} & \\
\times & \times \prod_{i=1}^{m}\left[\left\{\prod_{j=1}^{n_{i}} \lambda\left(t_{i j}\right)\right\} \int \ldots \int \exp \left\{-\int_{B_{i}(v)} \lambda(t) \mathrm{d} \mathbf{x} \mathrm{d} t\right\} \mathrm{d} \mathbf{x}_{i 1} \cdots \mathrm{d} \mathbf{x}_{i n_{i}}\right],
\end{aligned}
$$

where

$$
A_{i}(v)= \begin{cases}{[0, W]^{d}} & \text { if } n_{i}=1, \\ \left\{\left(\mathbf{x}_{i 1}, \ldots, \mathbf{x}_{i n_{i}}\right): v \leq \frac{\| \mathbf{x}_{i k}-\mathbf{x}_{i l} \mid}{\left|t_{i k}-t_{i l}\right|}, 1 \leq k, l \leq n_{i}, \mathbf{x}_{i j} \in[0, W]^{d}\right\} & \text { if } n_{i}>1,\end{cases}
$$


represents possible germination locations $\left(\mathbf{x}_{i 1}, \ldots, \mathbf{x}_{i n_{i}}\right)$ that may correspond to the observed germination times assuming that the growth speed is $v$.

Example 2. Consider the same $\lambda(\cdot)=\alpha \mathbf{1}_{[a, b]}(\cdot)$ as in Example 1. By the same argument, the maximum likelihood estimators $\widehat{a}$ and $\widehat{b}$ of the parameters $a$ and $b$ are the minimum and the maximum of $\left\{t_{i j}: 1 \leq j \leq n_{i}, i=1, \ldots, m\right\}$, respectively. Thus, we can consider

$$
\begin{aligned}
& L_{2}(\alpha, v)=\left[\exp \left\{-W^{d} \alpha(b-a)\right\}\right]^{n-m} \alpha^{\sum_{i=1}^{m} n_{i}} \\
& \times \prod_{i=1}^{m}\left[\int \ldots \int \exp \left\{-\int_{B_{i}(v)} \alpha \mathbf{1}_{[a, b]}(t) \mathrm{d} \mathbf{x} \mathrm{d} t\right\} \mathrm{d} \mathbf{x}_{i 1} \cdots \mathrm{d} \mathbf{x}_{i n_{i}}\right] .
\end{aligned}
$$

Since maximising $\log L_{2}(\alpha, v)$ is analytically intractable, numerical optimisation may be adopted.

\section{Simulation}

A series of simulations with the intensity given in equation (2.1) was done to assess the quality of such maximum likelihood estimation. The values of $v, \alpha, \gamma$ and $k$ chosen were similar to those considered in Chiu et al. (2000) and Molchanov and Chiu (2000). For $d=1$, we chose $k=1$ and $k=2$ because the shapes of the intensity functions differ substantially. For each set of parameter values, $n=100$ independent realisations have been simulated 50 times.

Because analytical optimisation is not feasible, we sketched the log-likelihood surface by calculating the log-likelihood values on a lattice of $v, \alpha, \gamma$ and $k$. The integrals are approximated by Monte-Carlo method. The results are reported in Table 1 .

We can see that the means are quite close to the true parameter values. Note that, by definition, the estimates of $v$ based on times and locations always over-estimate the true speed, but the biases are only about $1 \%$ of the true value; their standard deviations are very 
Table 1: Means and standard deviations of the maximum likelihood estimates obtained from 50 replicates of 100 independent realisations.

\begin{tabular}{lcccc}
\hline \multicolumn{1}{c}{$\widehat{\alpha}$} & $\widehat{\gamma}$ & $\widehat{k}$ & $\widehat{v}$ \\
\hline \hline$d=1, \alpha=6, \gamma=1, k=2, v=0.2$ & & & \\
\hline times and locations & $6.069(0.598)$ & $1.004(0.174)$ & $2.008(0.175)$ & $0.202(0.002)$ \\
times only & $5.912(1.048)$ & $1.080(0.273)$ & $2.036(0.199)$ & $0.192(0.031)$ \\
\hline$d=1, \alpha=6, \gamma=1, k=1, v=0.2$ & & & \\
\hline times and locations & $6.106(0.600)$ & $1.002(0.185)$ & $1.000(0.071)$ & $0.202(0.002)$ \\
times only & $5.738(0.821)$ & $1.150(0.261)$ & $1.016(0.087)$ & $0.183(0.036)$ \\
\hline$d=2, \alpha=6, \gamma=1, k=2, v=0.2$ & & & \\
\hline times and locations & $5.988(0.400)$ & $1.054(0.115)$ & $2.060(0.118)$ & $0.201(0.001)$ \\
times only & $6.026(0.654)$ & $1.020(0.170)$ & $2.012(0.162)$ & $0.193(0.022)$ \\
\hline
\end{tabular}

small, compared with those of other parameters or with those of the estimates of $v$ based on times only. The standard deviations of the estimates of $v$, no matter whether they are based on times and locations or on times only, are much smaller than those of the other estimates. For $d=1$, the biases and the standard deviations of the estimates based on times and locations are smaller than those based on times only. Increasing the dimension from one to two does not lead to poorer estimates.

\section{Neurobiological Data}

We consider the autoinhibited release of neurotransmitters at a synapse as described in Section 1.

The positive release time (germination time) values ranged up to 1000 so we divided them 
by 500 so as to get roughly the range of time values we encountered in the simulations. The minimum, maximum, mean and standard deviation of the normalised first release times in the 699 experiments with at least one release (germination) are 1.036, 2.366, 1.329 and 0.129, respectively. Thus, there may be a delay of $\tau$ time units in the release of neurotransmitters. Following Chiu et al. (2000), we assumed that $\tau=1, W=1$, and adopted the unnormalised gamma density suggested by Thomson et al. (1995). Consider that the intensity is given by

$$
\Lambda(t)=\alpha \mathbf{1}_{[\tau, \infty)}(t) \int_{\tau}^{t} \frac{\gamma^{k}}{\Gamma(k)}(s-\tau)^{k-1} e^{-\gamma(s-\tau)} \mathrm{d} s .
$$

The aim of this case study is to estimate the parameters in (6.1).

First, we used the release times in the 800 experiments to estimate the parameters in (6.1). The log-likelihood surface in the $(\alpha, \gamma, k, v)$-space was sketched on a rough lattice $[0.8,2.5] \times[1,25] \times[3.5,15] \times[0.01,0.5]($ mesh sizes $=0.2,0.5,0.15$ and 0.04 , respectively $)$. See Figure 1. Then we used a smaller and finer lattice to get a better resolution in the neighbourhood of the maximum. After several iterations we got $(1.5,11.6,4.9,0.24)$ from the lattice $[0.8,2] \times[10,13] \times[4,6] \times[0.08,0.3]($ mesh sizes $=0.1,0.2,0.1$ and 0.01 , respectively $)$ as the maximum likelihood estimates, and the log-likelihood surface projected on to the four axes is given in Figure 2.

Second, we followed Chiu et al. (2000) and took the inverse square root transformation of the amplitude to be the distance surrogate. There were 50 experiments with two identical amplitude readings. Since in our model it is impossible to have two germinated seeds at the same location, we ignored these experiments. Of the transformed data, there are four outliers above 0.2 (all with value 0.2234 ) so we deleted these experiments as well, leaving us with 101 experiments with no germinated seeds and 645 with at least one germinated seed, the frequencies of 1's, ..., 4's now being 387, 210, 45, 3. The location values were multiplied by 5 so they were roughly uniform on $[0,1]$. 

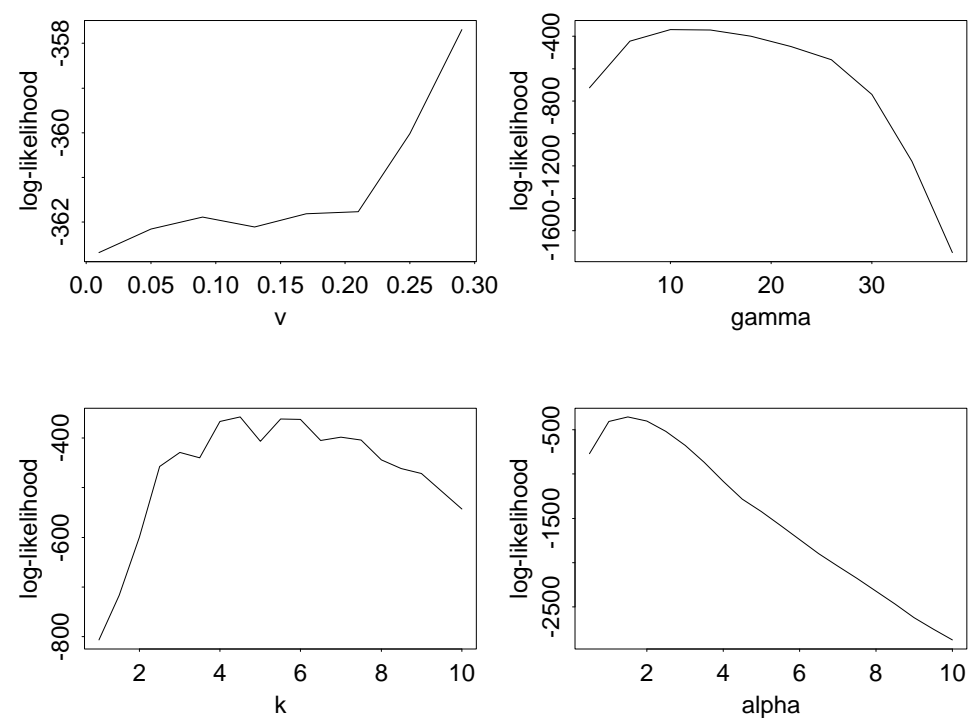

Figure 1: The log-likelihood surface projected on to the four axes of the lattice $[0.8,2.5] \times$ $[1,25] \times[3.5,15] \times[0.01,0.5]($ mesh sizes $=0.2,0.5,0.15$ and 0.04 , respectively $)$.
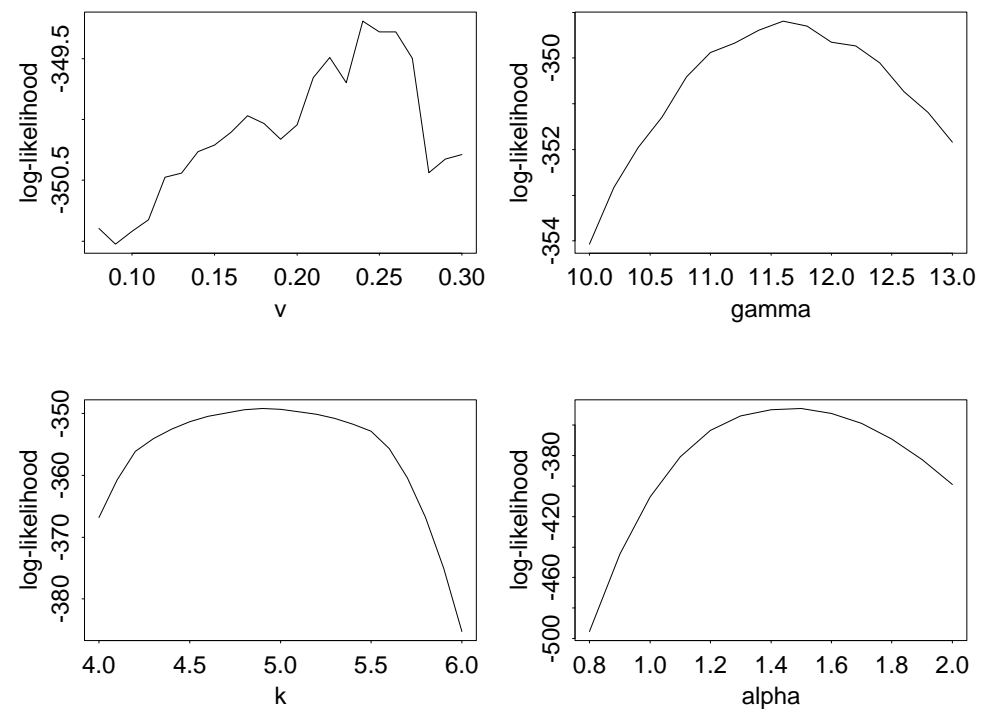

Figure 2: The log-likelihood surface projected on to the four axes of the lattice $[0.8,2] \times$ $[10,13] \times[4,6] \times[0.08,0.3]($ mesh sizes $=0.1,0.2,0.1$ and 0.01 , respectively $)$. 
The maximum likelihood estimate of the speed is the same as in Chiu et al. (2000), i.e. 0.018. Using the same iteration procedure as described above, we got $(1.29,13.3,5.36)$ from the lattice $[1.2,1.4] \times[12,14.5] \times[5,6.7]($ mesh sizes $=0.01,0.05$ and 0.01 , respectively $)$ as the maximum likelihood estimates of $(\alpha, \gamma, k)$.

We can see from the summary given in Table 2 that the maximum likelihood estimates of $\alpha, \gamma$ and $k$ based on release times only are close to those based on release times and transformed amplitudes, which are also close to the estimates obtained in Chiu et al. (2000). However, the estimates of the speed are very different. The reason is that, although we deleted 50 experiments with identical amplitudes, there are still experiments with very similar amplitudes, and so if the transformed amplitudes were regarded as locations, then the speed had to be very small. Such a substantial difference in these two estimates of $v$ implies that the inverse square root transformation of the amplitude should not serve as location surrogate. Table 2 also reports the estimates obtained by Thomson (see Holst, Quine and Robinson, 1996, p. 921), who suggested on biological grounds that $k=5$, and used the number of experiments with zero releases and the times of the first two releases to estimate $\alpha, \gamma$ and $v$, based on another series of 800 experiments. Since the conditions of the experiments were the same, the estimates should be comparable. We observe that all the estimates of $k$ given in this paper and Chiu et al. (2000) are very close to the value suggested on biological grounds.

\section{Acknowledgements}

SNC and ISM were partially supported by the UK/Hong Kong Joint Research Scheme and the UK Engineering and Physical Sciences Research Council, and SNC and MPQ were partially supported by the Research Grants Council of the Hong Kong Special Administrative Region, China (Project no. HKBU/2075/98P) and an FRG grant of the Hong Kong Baptist University. 
Table 2: Estimates for neurobiological data.

\begin{tabular}{lcccc}
\hline & $\widehat{\alpha}$ & $\widehat{\gamma}$ & $\widehat{k}$ & $\widehat{v}$ \\
\hline Thomson* (see Holst et al., 1996, p.921) & 2.04 & 2.94 & $5^{\dagger}$ & 0.20 \\
Chiu et al. (2000) & 1.590 & 13.296 & 5.100 & 0.018 \\
MLE based on times and amplitudes & 1.29 & 13.3 & 5.36 & 0.018 \\
MLE based on times only & 1.5 & 11.6 & 4.9 & 0.24 \\
\hline
\end{tabular}

* These estimates were obtained from another series of 800 experiments.

$\dagger$ The value 5 was suggested on biological grounds.

\section{References}

Bennett, M. R. and Robinson, J. (1990) Probabilistic secretion of quanta from nerve terminals at synaptic sites on muscle cells: nonuniformity, autoinhibition and the binomial hypothesis. Proceedings of the Royal Society of London, Series B 239, 329-358.

Chiu, S. N. (1995) Limit theorems for the time of completion of Johnson-Mehl tessellations. Advances in Applied Probability 27, 889-910.

Chiu, S. N. (1997) A central limit theorem for linear Kolmogorov's birth-growth models. Stochastic Processes and their Applications 66 97-106.

Chiu, S. N. and Quine, M. P. (1997) Central limit theory for the number of seeds in a growth model in $\mathbb{R}^{d}$ with inhomogeneous Poisson arrivals. Annals of Applied Probability $7,802-814$.

Chiu, S. N., Quine, M. P. and Stewart, M. (2000) Nonparametric and parametric estimation for a linear germination-growth model. Biometrics 56, 755-760.

Cowan, R., Chiu, S. N. and Holst, L. (1995) A limit theorem for the replication time of a 
DNA molecule. Journal of Applied Probability 32, 296-303.

Holst, L., Quine, M. P. and Robinson, J. (1996) A general stochastic model for nucleation and linear growth. Annals of Applied Probability 6, 903-921.

Johnson, W. A. and R. F. Mehl (1939) Reaction kinetics in processes of nucleation and growth. Transaction of the American Institute of Mining, Metallurgical and Petroleum Engineers 135, 416-458.

Kolmogorov, A. N. (1937) On statistical theory of metal crystallisation. Izvestia Academii Nauk SSSR, Seria Matematicheskaya 3, 355-360.

Molchanov, I. S. and Chiu, S. N. (2000) Smoothing techniques and estimation methods for non-stationary Boolean models with applications to coverage processes. Biometrika $\mathbf{8 7}$ 263-283.

Okabe, A., Boots, B., Sugihara, K. and Chiu, S. N. (2000) Spatial Tessellations. Concepts and Applications of Voronoi Diagrams, Second edition. Chichester, U.K.: John Wiley \& Sons.

Quine, M. P. and Robinson, J. (1992). Estimation for a linear growth model. Statistics and Probability Letters 15, 293-297.

Thomson, P. C., Lavidis, N. A., Robinson, J. and Bennett, M. R. (1995). Probabilistic secretion of quanta at somatic motor-nerve terminals: the fusion pore model, quantal detection and autoinhibition. Philosophical Transactions of the Royal Society of London. Series B 349, 197-214.

Vanderbei, R. J. and Shepp, L. A. (1988) A probabilistic model for the time to unravel a strand of DNA. Stochastic Models 4, 299-314.

Wolk, C. P. (1975). Formation of one-dimensional patterns by stochastic processes and by filamentous blue-green algae. Developmental Biology 46, 370-382. 